\title{
Factors Affecting the Detection Rate of Human Papillomavirus
}

Diane M. Harper, MD, MPH',

Megban R. Longacre, $P b D^{1}$

Walter W. Noll, $M D^{3}$

Dorotby R. Belloni, BS

Bernard F. Cole, $P b D^{2}$

'Department of Obstetrics and Gynecology, Dartmouth Medical School, Hanover, NH

${ }^{2}$ Department of Community and Family Medicine, Dartmouth Medical School, Hanover, $\mathrm{NH}$

${ }^{3}$ Department of Pathology, Dartmouth Medical School, Hanover, $\mathrm{NH}$

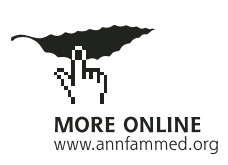

\begin{abstract}
BACKGROUND Maximizing the accuracy of human papillomavirus (HPV) detection from a single sample is important for clinical and research purposes. The purpose of this study was to determine whether cyclic hormonal variation, recent sexual intercourse, interval between samplings, and the technique used to sample affect the detection of HPV.
\end{abstract}

METHODS This study was a prospective, longitudinal, randomized controlled trial. Three techniques for self-sampling (2 consecutive synthetic polyester fiber [Dacron] swabs, a single Dacron swab, and a tampon) were repeated at 3 different sampling times during a period of 4 to 6 weeks in addition to 1 cliniciandirected sampling of the ectocervix and endocervix at the first sampling time. All self-samplings were taken in a proscribed randomized order. Women (aged 18 to 68 years) attending a colposcopy clinic for abnormal cytology or abnormal cervical appearance participated in the study. The outcome measure was the detection of HPV by polymerase chain reaction amplification.

RESULTS The 103 participants provided 1,189 cervicovaginal samplings. Logistic regression indicated that intercourse within 48 hours of sampling did not result in a greater detection of high-risk or any HPV type (odds ratio [OR] $=1.05,95 \%$ confidence interval $[\mathrm{Cl}], 0.65-1.69 ; \mathrm{OR}=1.08,95 \% \mathrm{Cl}, 0.73-1.60$, respectively). Among those women who have regular menstrual cycles, there was no cyclic effect on HPV detection for high-risk and any HPV types. Time from previous sampling did not affect HPV detection. Among the self-sampling techniques, using a single self-swab and the tampon resulted in the detection of HPV between $10 \%$ and $35 \%$ less often than using 2 consecutive swabs $(P<.025)$. Self-sampling with 2 swabs was not significantly different from clinician sampling for detecting high-risk HPV types (OR for self-sampling $=0.87(95 \% \mathrm{Cl}, 0.66-1.13)$ ).

CONCLUSIONS HPV detection is not dependent on menstrual cycle timings, the recency of intercourse, or the time between samplings, but it is dependent on the sampling technique.

Ann Fam Med 2003;1:221-227. DOI: 10.1370/afm.90.

\section{INTRODUCTION}

$\mathrm{H}$ uman papillomavirus (HPV) testing plays a critical role in clinical evaluation of abnormal Papanicolaou (Pap) smears and in cervical cancer research. Point detection of high-risk HPV DNA is the outcome measure of many cervical cancer screening studies, including those about HPV vaccines, ${ }^{1}$ primary HPV screening ${ }^{2-8}$ triage after an atypical squamous cells of undetermined significance (ASCUS) Pap test finding, ${ }^{9}$ and HPV persistence. ${ }^{10-12} \mathrm{HPV}$ testing is used to detect a sexually transmitted infection. Point prevalence of any HPV type - in contrast to only high-risk types - is the outcome measure of studies of immune response modifiers and HPV clearance. ${ }^{13}$ There are clinical and research scenarios for which a self-administered technique would be the preferred method of sampling for HPV. Self-sampling has been shown to be equivalent to
Diane M. Harper, MD, MPH, MS Dartmouth-Hitchcock Medical Center One Medical Center Drive Lebanon, NH 03756

Diane.M.Harper@Dartmouth.edu 
clinician-directed sampling, , $^{2,14-16}$ is technically feasible with mailed return samples ${ }^{14,17}$ and is being used programmatically to screen women for sexually transmitted infections in Australia and Papua New Guinea. ${ }^{28-27}$

The cumulative presence of HPV is always greater than its point prevalence, suggesting that single-point sampling is less than $100 \%$ sensitive. Because clinical management and research usually depend on singlepoint detection of HPV, it is important to understand factors that contribute to false-negative and false-positive results. We hypothesized that HPV detection could be dependent on at least 4 factors: hormonal fluctuations in the menstrual cycle, the recency of unprotected vaginal intercourse, the frequency of repeated sampling, and the sampling technique. The primary objective of our study was to test the influence of 4 predictors on the detection of HPV in women who self-sampled during a 4- to 6-week period. Our secondary objective was to determine the relation of these hypothesized influences with the traditionally linked HPV risk factors of age, sexual practice, contraceptive method, and tobacco use.

\section{METHODS}

\section{Study Population}

The participants in this longitudinal randomized controlled trial were women seen in 3 residency-based colposcopy clinics who were 18 or older, not pregnant, and had been referred for colposcopy because of an abnormal Pap smear result or an abnormal finding on a visual cervical examination. The Committee for the Protection of Human Subjects at Dartmouth College approved this study. All participants were enrolled at the time of the colposcopy visit, and consent forms and questionnaires were completed before the colposcopic examination. Of the 156 women invited to take part in the study, 103 (66\%) agreed to participate. The other 53 declined because of uneasiness about the upcoming colposcopic examination.

\section{Initial Data Collection}

HPV detection using different sampling techniques was performed before the colposcopic examination or biopsy acquisition. Each participant engaged in $5 \mathrm{HPV}$ screening tests at the initial visit, using the following techniques: clinician-directed ectocervical swab, clinician-directed endocervical swab, a self-sampled synthetic polyester fiber swab (Dacron), a second self-sampled Dacron swab, and a self-sampled tampon, performed in random order. The order of sampling, clinician vs self, was randomized first. The order of the self-sampling techniques, Dacron swabs vs tampons, was randomized second. This process produced 4 possible orders of sampling at the colposcopy visit. None of the sampling techniques caused visible cervical or vaginal bleeding. Both written and verbal instructions for self-sampling were provided for the participants. More detailed descriptions of using the tampons for self-sampling have been published. ${ }^{14,16}$

\section{Subsequent Data Collection}

Each week thereafter, until 3 more sets of samples had been obtained, participants performed home sampling with the Dacron swabs and tampon. All participants used the same random order of self-sampling techniques at each sampling as was determined at the colposcopy visit. For example, a woman randomized to "self-tampon, self-swabs, clinician" at the colposcopy visit would self-sample at home with the tampon first, then use the 2 Dacron swabs consecutively for each of the next 3 sampling periods. If menses occurred during a scheduled sampling time, participants were asked not to self-sample, but to resume the collections as soon as possible after menses ended, until 3 collections were completed. The project manager attempted to remind each woman the night before her scheduled sampling. Three samples (2 Dacron swabs and 1 tampon) were placed in individual tubes with PreservCyt (Cytyc, Boxborough, Mass) as the transport medium at each sampling time.

PreservCyt is stable within a wide temperature range $\left(-4^{\circ}\right.$ to $\left.37^{\circ} \mathrm{C}\right)$ and preserves cells for up to 4 weeks. Each set of samples was placed in a biohazard bag and mailed back to the Dartmouth HPV laboratory for immediate processing.

\section{HPV DNA Analysis}

The laboratory processing and HPV detection method have been described. ${ }^{14}$ Briefly, the Dartmouth-Hitchcock Medical Center Molecular Diagnostic Laboratory performed all HPV tests with internal and external controls. A maximum of 18 high-risk HPV types (16, 18, 26, 31, 33, 35, 39, 45, 51, 52, 55, 56, 58, 59, 68, MM4, MM7, MM9) and 9 low-risk HPV types $(6,11$, $40,42,53,54,57,66, \mathrm{MM} 8)$ could be detected individually by polymerase chain reaction (PCR) amplification using the MY09-MY11-HMB01 L1 consensus primer system and a reverse line blot detection strip (Roche Molecular Systems, Alameda, Calif). Excessively bloody samples could provide enough hemoglobin to inhibit the PCR process used; therefore, sampling during menses was avoided.

\section{Diary and Descriptive Data}

During the home-sampling period, participants were asked to keep diaries of their menstrual bleeding, when 
they self-sampled, and when they had intercourse. If they had more than 1 partner, participants were asked to indicate in a nonidentifying manner the dates of intercourse with the different partners. The diaries provided the data to evaluate HPV detection in relation to the menstrual cycle and intercourse within 48 hours before self-sampling. They also provided data to evaluate whether the number of days from a previous sampling would affect the HPV detection rate.

Other demographic and medical information gathered included age, pregnancy history, smoking history, participants' cytologic findings when first examined, histologic diagnosis from colposcopic testing, and use of hormonal and barrier contraceptive methods.

\section{Data Recombination}

Fourteen unique specimens for each woman were collected ( 2 clinician-directed swabs, 2 self-directed swabs, and 1 tampon from the initial clinician visit, and 2 swabs and 1 tampon for the subsequent 3 home samplings). Based on our previous results, ${ }_{1}^{14}$ we statistically combined the clinician-directed ectocervical and endocervical samplings into 1 clinician-directed result per woman. The combined clinician-directed result was considered the referent level against which self-sampling was compared for the logistic regressions. We statistically combined the 2 Dacron swabs from each of the self-samplings into a double-swab result, maintaining the first single swab as a unique result in this analysis. The second swab result was used only to provide the double-swab result and was omitted as a second single unique result. In summary, the samplings for HPV detection were the clinician-directed technique, the double self-swab technique, the single self-swab technique, and the self-tampon technique, for a total of 4 techniques at the initial visit, and 3 at each of 3 subsequent samplings.

\section{Statistics}

This study was powered at $82 \%$ to detect, between 2 sampling devices, a $10 \%$ discordant pair rate and a difference in HPV detection rates of $8 \%$ by a 2 -tailed McNemar test at a .05 level of significance. Logistic regression models were used in a univariate and multivariate manner to determine the effect of the study variables on the detection of high-risk and any types of HPV. Because our study sample comes from a clustered design, and because there is a high correlation within the repeated measures of each variable, the logistic regression models were modified by using generalized estimating equations to extend the generalized linear models with an exchangeable correlation matrix for the sampling structure. ${ }^{32}$

\section{RESULTS}

\section{Sample Characteristics}

The 103 participants in the study were an average age of 37.7 years (range of 18 to 68 years) and had a mean of 2.3 pregnancies (SD 1.9) and 1.5 births (SD 1.3). Within the past 3 years, $64 \%$ had 1 sexual partner, $19.4 \%$ had 2 sexual partners; and $16.6 \%$ had 3 or more sexual partners. Eighteen percent were current tobacco users, and $34 \%$ were previous users. More than one half of the women, $54.9 \%$, used no hormonal or barrier contraceptive, $20.4 \%$ were currently using and $64.1 \%$ had ever used a combination oral contraceptive, $10.8 \%$ used a barrier method (mostly condoms and foam), 7.8\% used an intrauterine device, and $4.9 \%$ used progesterone-only contraceptives. Of the participants, $38.8 \%$ engaged in intercourse within 48 hours before at least 1 of the self-samplings. There was an average of 9.7 days (SD 7.6) between samplings. On at least 1 of the 14 samples, $43.7 \%(n=45)$ of the participants were positive for high-risk types of $\mathrm{HPV}_{;} 49.5 \%(\mathrm{n}=51)$ were positive for any (high-risk or low-risk) HPV, and 54.6\% of all women $(\mathrm{n}=56)$ had cervical intraepithelial neoplasia (CIN) described on a histology report.

Of 1,339 possible samples for HPV, 1,186 were returned and registered for this analysis, for a return rate of $88.6 \%$. The swab and tampon samples not returned were very often missing from the same woman. Approximately 35\% of the samples were taken at the first visit, with another $20 \%$ to $22 \%$ at each of the 3 home samplings. The distribution of samples by week within the menstrual cycle ranged from $15 \%$ (weeks 1 and 4) to 25\% (weeks 2 and 5). Most, 69\%, of the samples were taken within 7 days of the previous sampling; 24\% were taken between 8 and 14 days of the previous sampling, and $7 \%$ were taken more than 14 days from the previous sampling. Overall, 26.7\% of the samples were positive for high-risk types of HPV, and $41.3 \%$ were positive for any HPV. The percentage of samples, by week, positive for high-risk and any type of HPV are displayed in a supplementary table (http:// www.annfammed.org/cgi/content/full/1/4/221/DC1). As shown in this table, the percentage of samples positive for HPV did not differ by week of the menstrual cycle, nor did it differ by time between samplings.

At the initial visit all 103 women provided 4 samples each; $91(88.3 \%)$ women provided all 3 samples each at the second week (12 women did not provide either swab or tampon at this sampling) $88(85.4 \%)$ women provided all 3 samples each at the third week ( 1 woman contributed samples at this visit where she had not at the second week, and 4 additional women did not contribute samples at the third week); and $81(78.6 \%)$ 
women completed all 3 samples each, and 1 woman completed just 1 sample (tampon) at the fourth week (6 additional women did not contribute any samples). Three women whose test results were positive for any HPV at the initial visit had test results that were subsequently negative for any HPV on all follow-up samplings, giving a less than $3 \%$ clearance rate of HPV after enrollment. One woman whose test result was negative for any HPV at the initial visit had a positive result for the same high-risk type at the subsequent 3 visits.

\section{Detection of High-Risk HPV Types}

Univariate logistic regression analysis was used to determine the crude odds ratios for each of the possible predictors for detecting high-risk HPV types. Table 1 shows that women who were older, who had more pregnancies and live births, and who had fewer than 14 days from a previous self-sampling had significantly fewer cases of high-risk HPV detected than their counterparts. Women who used combined oral contraceptives, whose cytology report was low-grade squamous intraepithelial lesion (LSIL) or high-grade squamous intraepithelial lesion (HSIL), or whose pathology report was any cervical intraepithelial neoplasia (CIN 1,2 , or 3) were more likely to have high-risk HPV detected on their samples. Tobacco use, other contraceptive methods, (including Depo-Provera or Norplant use and barrier methods), the order of sampling, the

Table 1. Crude Odds Ratios of Predictors of Detection of Human Papillomavirus (HPV)

\begin{tabular}{|c|c|c|c|c|c|c|c|}
\hline \multirow[b]{2}{*}{ Variable } & \multirow[b]{2}{*}{ No. } & \multirow[b]{2}{*}{ Reference } & \multirow[b]{2}{*}{ Levels } & \multicolumn{2}{|c|}{ High-Risk HPV } & \multicolumn{2}{|l|}{ Any HPV } \\
\hline & & & & OR $(95 \% \mathrm{Cl})$ & P Value & OR $(95 \% \mathrm{Cl})$ & P Value \\
\hline Age & 1,186 & Young age & Older age & $0.96(0.93-0.99)$ & .014 & $0.94(0.91-0.98)$ & .001 \\
\hline Gravidity & 1,177 & No pregnancies & $\begin{array}{l}\text { One or more } \\
\text { pregnancies }\end{array}$ & $0.81(0.65-1.00)$ & .05 & $0.80(0.64-0.99)$ & .039 \\
\hline Parity & 1,171 & No births & $\begin{array}{l}\text { One or more } \\
\text { births }\end{array}$ & $0.66(0.48-0.92)$ & .013 & $0.58(0.42-0.81)$ & .002 \\
\hline Oral contraceptive use & 1,177 & No & Yes & $2.55(1.10-5.86)$ & .028 & $2.17(0.90-5.22)$ & NS \\
\hline Depo-Provera or Norplant & 1,177 & No & Yes & $1.28(0.31-5.32)$ & NS & $2.01(0.32-12.61)$ & NS \\
\hline Barrier methods & 1,177 & No & Yes & $1.16(0.42-3.22)$ & NS & $1.15(0.36-3.68)$ & NS \\
\hline Intrauterine device* & 1,182 & No & Yes & $0.12(0.02-0.99)$ & .049 & & \\
\hline \multirow[t]{4}{*}{ Cytology } & 1,186 & Normal & ASCUS & $2.14(0.62-7.33)$ & NS & $0.56(0.16-2.02)$ & NS \\
\hline & & & LSIL & $7.56(2.14-26.70)$ & .002 & $3.09(0.75-12.7)$ & NS \\
\hline & & & HSIL & $13.47(3.08-58.89)$ & .001 & $3.66(0.71-18.83)$ & NS \\
\hline & & & AGUS & $1.30(0.18-9.48)$ & NS & $1.42(0.07-29.09)$ & NS \\
\hline \multirow[t]{2}{*}{ Pathology } & 1,186 & Normal & CIN 1 & $2.87(1.29-6.37)$ & .010 & $2.53(1.16-5.53)$ & .020 \\
\hline & & & $\operatorname{CIN} 2 / 3$ & $13.46(3.15-57.60)$ & $<.001$ & $7.10(1.37-36.90)$ & .020 \\
\hline Tobacco use ${ }^{\dagger}$ & 1,177 & No & Yes & $1.01(0.48-2.11)$ & NS & $0.88(0.42-1.84)$ & NS \\
\hline $\begin{array}{l}\text { Intercourse within } 48 \text { hours } \\
\text { of sampling }\end{array}$ & 642 & No & Yes & $1.05(0.65-1.68)$ & NS & $1.07(0.73-1.55)$ & NS \\
\hline $\begin{array}{l}\text { Randomization of person } \\
\text { collecting sample: clinician } \\
\text { or self }\end{array}$ & 408 & Self & Clinician & $0.59(0.14-2.45)$ & NS & $0.51(0.12-2.18)$ & NS \\
\hline $\begin{array}{l}\text { Randomization of technique } \\
\text { used first: swab or tampon }\end{array}$ & 1,186 & Swab & Tampon & $1.28(0.60-2.74)$ & NS & $1.92(0.89-4.14)$ & NS \\
\hline $\begin{array}{l}\text { Number of days from } \\
\text { previous sampling }\end{array}$ & 1,186 & 15 or more days & $1-14$ days & $0.82(0.66-1.00)$ & .055 & $0.85(0.70-1.04)$ & NS \\
\hline \multirow{4}{*}{$\begin{array}{l}\text { Sample taken during specific } \\
\text { week of menstrual cycle }\end{array}$} & 1,138 & Week 1 & Week 2 & $0.98(0.63-1.52)$ & NS & $1.00(0.76-1.31)$ & NS \\
\hline & & & Week 3 & $1.04(0.63-1.72)$ & NS & $1.08(0.80-1.46)$ & NS \\
\hline & & & Week 4 & $1.17(0.72-1.93)$ & NS & $1.14(0.80-1.62)$ & NS \\
\hline & & & Week 5 & $0.80(0.47-1.36)$ & NS & $0.79(0.52-1.22)$ & NS \\
\hline \multirow{3}{*}{$\begin{array}{l}\text { Clinician double swab vs } 2 \\
\text { consecutive self-swabs vs } \\
\text { single self-swab vs tampon }\end{array}$} & 1,184 & Clinician-directed & 2 consecutive & $0.81(0.63-1.03)$ & NS & $0.84(0.73-0.96)$ & .010 \\
\hline & & cervical swabs & 1 self-swab & $0.66(0.51-0.87)$ & .003 & $0.84(0.73-0.96)$ & .010 \\
\hline & & & Tampon & $0.61(0.46-0.81)$ & .001 & $0.85(0.74-0.96)$ & .013 \\
\hline \multicolumn{8}{|c|}{$\begin{array}{l}\text { OR = odds ratio; } \mathrm{Cl}=\text { confidence interval; } \mathrm{ASCUS}=\text { abnormal squamous cells of undetermined significance; NS = not significant; LSIL = low-grade squamous intraep- } \\
\text { ithelial lesion; } \mathrm{HSIL}=\text { high-grade squamous intraepithelial lesion, } \mathrm{CIN}=\text { cervical intraepithelial neoplasia. } \\
\text { * There are insufficient numbers of samples associated with women using an intrauterine device who also had high-risk types of HPV to include the IUD in the logistic } \\
\text { regression. } \\
+ \text { Tobacco use is current or ever vs never. } \\
\text { f Weeks are defined as } 0-7,8-14,15-21,22-35 \text {, and } 36+\text { days. Week } 5 \text { encompasses women with long menstrual cycles, using progesterone only contraceptives, or who } \\
\text { are postmenopausal. }\end{array}$} \\
\hline
\end{tabular}


weeks of the menstrual cycle, and intercourse within 48 hours before self-sampling did not predict the detection of high-risk HPV types. No woman noted any change of sexual partners in her diary during the sampling interval.

The detection of high-risk HPV from the self-sampling techniques of 2 swabs, $1 \mathrm{swab}$, and 1 tampon was compared with detection from the clinician-directed technique. The single swab and tampon detected fewer cases of high-risk HPV than the clinician technique; and the self-sample double-swab technique appeared to be equivalent to the clinician double-swab technique (odds ratio $[\mathrm{OR}]=0.81,95 \%$ confidence interval $[\mathrm{CI}]$, $0.63-1.03)$.

All variables listed in Table 1 were included in the multivariate logistic regressions to ensure that there was no negative confounding by variables with nonsignificant crude odds ratios. The adjusted odds ratios for the variables of interest and the traditional risk factors indicated that gravidity, cytology, and pathology remained significant predictors of high-risk HPV. All 4 study variables of interest were not significant predictors of HPV detection except for the single-swab and tampon techniques that less frequently detected high-risk HPV. Table 2 highlights the adjusted odds ratios for the variables of interest in this study. The single swab and the tampon were about $30 \%$ less likely to detect high-risk HPV than the clinician-directed swabs. The doubleswab method was equivalent to clinician-directed sampling $(\mathrm{OR}=0.87,95 \% \mathrm{CI}, 0.66-1.13)$. Intercourse within 48 hours before sampling did not affect the detection of high-risk HPV. The number of days between sampling and the week of the menstrual cycle did not affect the detection of high-risk HPV.

\section{Detection of Any HPV Types}

Table 1 shows that predicting the detection of any type of HPV by univariate logistic regression was similar to predicting the detection of high-risk HPV types. The single swab and tampon detected HPV less frequently than the double-swab clinician technique, and the self-sample double-swab technique appeared to be equivalent to the clinician double-swab technique. Multivariate logistic regressions with all variables and combinations of variables showed that findings for detecting any type of HPV were similar to findings for detecting only high-risk types. Table 2 shows that all self-sampling methods are about $8 \%$ less likely to detect any type of HPV than the clinician-directed swabs, except the 2 self-swabs, which had a detection rate that was not statistically different from the clinician-directed swab rate. Recent intercourse, the number of days between samplings, and the week of the menstrual cycle did not affect the detection of any type of HPV.

\section{DISCUSSION}

Self-sampling for HPV is becoming a recognized method for epidemiologic studies, as well as a clinical tool that is inexpensive, feasible, and viewed by women as convenient and acceptable. ${ }^{14}$ The limitation of any HPV sampling technique is the degree to which it is affected by the specific modifiable factors of timing and technique. The current study is the largest series of repeated samplings published to date in which timing in the menstrual cycle, timing after intercourse, timing after previous sampling, and technique used were evaluated independently and in context with the traditionally

Table 2. Adjusted Odds Ratios for Predictors of Human Papillomavirus (HPV) Detection

\begin{tabular}{|c|c|c|c|c|c|c|}
\hline \multirow[b]{2}{*}{ Variable* } & \multirow[b]{2}{*}{ Reference } & \multirow[b]{2}{*}{ Levels } & \multicolumn{2}{|c|}{ High-Risk HPV } & \multicolumn{2}{|c|}{ Any HPV } \\
\hline & & & OR $(95 \% \mathrm{CI})$ & P Value & OR $(95 \% \mathrm{Cl})$ & P Value \\
\hline $\begin{array}{l}\text { Intercourse within } \\
48 \text { hours of sampling }\end{array}$ & No & Yes & $1.05(0.65-1.69)$ & NS & $1.08(0.73-1.60)$ & NS \\
\hline $\begin{array}{l}\text { Number of days from } \\
\text { previous sampling }\end{array}$ & 15 or more days & $1-14$ days & $0.87(0.70-1.10)$ & NS & $0.85(0.69-1.05)$ & NS \\
\hline \multirow{4}{*}{$\begin{array}{l}\text { Sample taken during } \\
\text { specific week of } \\
\text { menstrual cycle }\end{array}$} & \multirow[t]{4}{*}{ Week 1} & Week 2 & $1.01(0.64-1.59)$ & NS & $1.02(0.77-1.35)$ & NS \\
\hline & & Week 3 & $1.08(0.65,1.81)$ & NS & $1.12(0.82-1.54)$ & NS \\
\hline & & Week 4 & $1.22(0.74-2.01)$ & NS & $1.18(0.81-1.70)$ & NS \\
\hline & & Week 5 & $0.81(0.48-1.38)$ & NS & $0.82(0.54-1.25)$ & NS \\
\hline \multirow[t]{3}{*}{ Technique } & \multirow{3}{*}{$\begin{array}{l}\text { Clinician-directed } \\
\text { ecto- and } \\
\text { endocervical swabs }\end{array}$} & $\begin{array}{l}2 \text { consecutive } \\
\text { self-swabs }\end{array}$ & $0.87(0.66-1.13)$ & NS & $0.91(0.85-0.98)$ & .015 \\
\hline & & 1 self-swab & $0.71(0.53-0.95)$ & .023 & $0.91(0.85-0.98)$ & .015 \\
\hline & & Tampon & $0.66(0.48-0.90)$ & .009 & $0.92(0.86-0.99)$ & .018 \\
\hline
\end{tabular}


described predictors of high-risk HPV. ${ }^{33-37}$ These particular 4 parameters were chosen because there are plausible scientific explanations for their effects. Hormonal fluctuation might affect HPV detection because progesterone causes deeper exfoliation of epithelium of the cervix and vagina, where HPV virion assembly takes place. Estrogen promotes cellular differentiation and exfoliation of only the superficial layers, which does not allow maturation of the parabasal cells that is required for HPV virion assembly and release. ${ }^{28}$ Previous work on the effect of cyclic hormones on HPV detection has not been conclusive. Of 2 representative studies, both using approximately 250 samples, 1 found HPV detection to be significantly higher in the progesterone-mediated luteal phase of the menstrual cycle, ${ }^{29}$ and the other found that HPV detection did not depend on the menstrual cycle, ${ }^{30}$ even though the cellular pellet size collected was increased during the second and third weeks, indicating increased exfoliation. ${ }^{31}$ We did not find an effect of the menstrual cycle phase on HPV detection.

Recent unprotected vaginal intercourse might affect HPV detection because false-positive tests could result from detecting another person's HPV DNA, or falsenegative tests could occur because vaginal penetration could mechanically remove HPV-infected cells. Our data show that intercourse within 48 hours before sampling, however, did not affect HPV detection rates.

Historically, cytologic testing for inadequate or abnormal cytology results was not repeated immediately for fear of a false-negative result. It was thought that the entire reservoir of potentially abnormal cells had been removed at the initial sampling. It is now recognized that frequently repeated cytologic testing does not result in false-negative results, and repeated cytology sampling at frequent intervals does not remove the entire reservoir of cells from which to sample (Gary Johnson, MD, oral communication about ASCUS and LSIL Triage Study results, August 11, 2003). This study did not show lower HPV detection rates with more frequent sampling for HPV. We found that the sampling technique did affect HPV detection. Self-sampling with 2 vaginal swabs was equivalent to clinician sampling with 2 cervical swabs and more sensitive than self-sampling with a single swab or a tampon.

The sampling technique could affect HPV detection in 3 ways. First, the vaginal epithelium occupies a much greater surface area than the cervical epithelium, and as such offers a greater number of potentially HPV-infected cells from which to sample. A self-sampling approach might not sample the cervix, but it will sample the vaginal epithelium, with a potentially greater likelihood of detecting HPV because of the larger area of potentially infected cells than the cervix offers. Second, HPV detection could be affected if the sampling technique does not release the cells for analysis. Cell clusters embedded in a Dacron swab or a rayon-covered cotton core tampon are not easily separated from the sampling device. It is expected that Dacron swabs will have more cells available for diagnosis when transported in the liquid cytology medium; despite collecting a small cellular pellet, our data suggest that they will release sufficient cells for reproducible results of HPV testing. ${ }^{7}$ Third, the sampling technique could affect HPV detection if too many cells were collected and released, thus inhibiting the PCR process. With tampons large cellular pellets result after vortexing. An excessive volume of DNA from these cellular pellets could inhibit the PCR reaction and could compromise the detection of HPV DNA. Our previous work, however, suggests that this explanation is unlikely for the reduced sensitivity of tampons reported here. We found tampons to be less sensitive than swabs (self- or clinician-directed) only when the tampon was used for less than 10 seconds. ${ }^{14}$ In contrast to the findings presented here, Fairley et $\mathrm{al}^{31}$ showed that tampons were equivalent to clinician-directed swabs.

HPV testing can serve as a test for sexually transmitted infection and as a cancer-screening test. As a test for a sexually transmitted infection, it will not be valuable until there is a pharmacological intervention to clear the anogenital HPV. As a cancer-screening test, testing for high-risk types of HPV serves as 1 of 3 triage methods for ASCUS cytology and as a test-of-cure for biopsyproven CIN 1 or CIN 2/3 lesions. ${ }^{38} \mathrm{HPV}$ testing in conjunction with cytology as a primary screening tool for cervical cancer has been approved for use in the United States. It is therefore important to establish now, before practice patterns are set, the most accurate and reliable methods to detect high-risk HPV types. On the basis of these data, cervical cancer screening programs can consider that a self-sampling technique using 2 consecutive swabs could be used as an alternative to clinician sampling to detect high-risk HPV.

To read commentaries or to post a response to this article, see the online version at http://www.annfammed.org/cgi/content/full/1/4/221.

Key words: Vaginal smears; menstrual cycle; coitus; tampons; swabs; papillomavirus; mass screening; delivery of health care; women's health

Submitted August 19, 2002; submitted, revised, August 11, 2003; accepted August 29, 2003.

Funding support: This study was supported by a grant from the Norris Cotton Cancer Center; Robert Wood Johnson Foundation (DMH), grant No. NCl-CA23108. Awarded 2002 Best Overall Prize Paper by the American Society of Colposcopy and Cervical Pathology at Biennial Meeting, Orlando, Florida, April 2002.

Acknowledgments: Patient recruitment: Debra Birenbaum, MD; Paul Hanissian, MD; Joan Barthold, MD; Sue Reilly, ARNP; Judith Tyson, MD; Ben Mahlab, MD; Laura Fry, MD; Cindy Rasmussen MD. Nursing coordinators: 
Julie Ackerman, LPN; Sheryle Hartwell, LPN. Research assistants: Erin Mulloy, Debie Thomas, MS. Study design consultant: Margaret Karagas, PhD. Review of pathology: Jorge Gonzalez, MD. HPV PCR reagents: Raymond J. Apple, PhD; Janet Kornegay, PhD, Roche Molecular Systems; Patti Gravitt, MS, National Cancer Institute. HPV PCR consultant: Cosette M. Wheeler, PhD, University of New Mexico. PreservCyt solutions: David J. Zahniser, PhD, Cytyc Corp. Illustrations for the patient education pamphlets: Gerald Auten, PhD.

\section{References}

1. Adams M, Borysiewicz L, Fiander $A$, et al. Clinical studies of human papilloma vaccines in pre-invasive and invasive cancer. Vaccine. 2001;19:2549-2556.

2. Wright TC Jr, Denny L, Kuhn L, Pollack A, Lorincz AT. HPV DNA testing of self-collected vaginal samples compared with cytologic screening to detect cervical cancer. JAMA. 2000;283:81-86.

3. Schiffman M, Herrero R, Hildesheim A, et al. HPV DNA testing in cervical cancer screening: results from women in a high-risk province of Costa Rica. JAMA. 2000;283:87-93

4. Cuzick J. Human papillomavirus testing for primary cervical cancer screening. JAMA. 2000;283:108-109.

5. Ratnam S, Franco EL, Ferenczy A. Human papillomavirus testing for primary screening of cervical cancer precursors. Cancer Epidemiol Biomarkers Prev. 2000;9:945-951.

6. Blumenthal PD, Gaffikin L, Chirenje ZM, McGrath J, Womack S, Shah $\mathrm{K}$. Adjunctive testing for cervical cancer in low resource settings with visual inspection, HPV, and the Pap smear. Int J Gynaecol Obstet. 2001;72:47-53.

7. Belinson J, Qiao Y, Pretorius R, et al. Prevalence of cervical cancer and feasibility of screening rural China: a pilot study for the Shanxi Province Cervical Cancer Screening Study. Int J Gynecol Cancer. 1999;9:411-417.

8. Womack SD, Chirenje ZM, Blumenthal PD, et al. Evaluation of a human papillomavirus assay in cervical screening in Zimbabwe. BJOG. 2000;107:33-38

9. Solomon D, Schiffman M, Tarone R, for the ALTS Study group. Comparison of three management strategies for patients with atypical squamous cells of undetermined significance: baseline results from a randomized trial. J Natl Cancer Inst. 2001;93:293-299.

10. Remmink AJ, Walboomers JM, Helmerhorst TJ, et al. The presence of persistent high-risk HPV genotypes in dysplastic cervical lesions is associated with progressive disease: natural history up to 36 months. Int J Cancer. 1995;61:306-311.

11. Ho GY, Burk RD, Klein S, et al. Persistent genital human papillomavirus infection as a risk factor for persistent cervical dysplasia. J Natl Cancer Inst. 1995;87:1365-1371.

12. Nobbenhuis MA, Walboomers JM, Helmerhorst TJ, et al. Relation of human papillomavirus status to cervical lesions and consequences for cervical-cancer screening: a prospective study. Lancet. 1999;354:20-25.

13. Tyring S. Imiquimod applied topically: a novel immune response modifier. Skin Therapy Lett. 2001;6:1-4.

14. Harper DM, Noll WW, Belloni DR, Cole BF. Randomized clinical trial of PCR-determined human papillomavirus detection methods: selfsampling versus clinician-directed-biologic concordance and women's preferences. Am J Obstet Gynecol. 2002;186:365-373.

15. Harper DM, Hildesheim A, Cobb JL, Greenberg M, Vaught J, Lorincz AT. Collection devices for human papillomavirus. J Fam Pract. 1999; 48:531-535.

16. Harper DM, Raymond M, Noll WW, Belloni DR, Duncan LT, Cole BF Tampon samplings with longer cervicovaginal cell exposures are equivalent to two consecutive swabs for the detection of high-risk human papillomavirus. Sex Transm Dis. 2002;29:628-636.

17. Ostergaard L, Moller JK, Andersen B, Olesen F. Diagnosis of urogenital Chlamydia trachomatis infection in women based on mailed sam ples obtained at home: multipractice comparative study. BMJ. 1996; 313:1186-1189.
18. Garland SM, Tabrizi SN, Fairley CK, Bowden FJ. Tampons could be used to diagnose sexually transmitted diseases. BMJ. 2001;322:676.

19. Gottlieb S. Tampons could be used to diagnose STDs. BMJ. 2000; 321:978.

20. Fairley CK, Chen S, Tabrizi SN, Quinn MA, McNeil JJ, Garland SM. Tampons: a novel patient-administered method for the assessment of genital human papillomavirus infection. J Infect Dis. 1992;165:1103-1106.

21. Tabrizi SN, Paterson B, Fairley CK, Bowden FJ, Garland SM. A selfadministered technique for the detection of sexually transmitted diseases in remote communities. J Infect Dis. 1997;176:289-292.

22. Tabrizi SN, Fairley CK, Cehn S, et al. Evaluation of patient-administered tampon specimens for Chlamydia trachomatis and Neisseria gonorrhoeae. Sex Transm Dis. 2000;27:133-137.

23. Paterson BA, Tabrizi SN, Garland SM, Fairley CK, Bowden FJ. The tampon test for trichomoniasis: a comparison between conventional methods and a polymerase chain reaction for Trichomonas vaginalis in women. Sex Transm Infect. 1998;74:136-139.

24. Bowden FJ, Paterson BA, Mein J, et al. Estimating the prevalence of Trichomonas vaginalis, Chlamydia trachomatis, Neisseria gonorrhoeae, and human papillomavirus infection in indigenous women in northern Australia. Sex Transm Infect. 1999;75:431-434.

25. Passey M, Mgone CS, Lupiwa S, et al. Community based study of sexual ly transmitted diseases in rural women in the highlands of Papua New Guinea: prevalence and risk factors. Sex Transm Infect. 1998;74:120-127.

26. Bowden FJ, Paterson BA, Garland SM, Tabrizi S, Fairley CK. Self administered tampons can be used to diagnose sexually transmitted diseases. BMJ. 1997;314:446.

27. Bowden FJ, Tabrizi SN, Paterson BA, Garland SM, Fairley CK. Determination of genital human papillomavirus genotypes in women in Northern Australia using a novel, self-administered tampon technique. Int J Gynecol Cancer. 1998;8:471-475.

28. Ferenczy A, Wright TC Jr. Anatomy and histology of the cervix. In: Kurman R, ed. Blaustein's Pathology of the Female Genital Tract. 5th ed New York, NY: Springer; 2002.

29. Schneider A, Kirchhoff T, Meinhardt G, Gissmann L. Repeated evaluation of human papillomavirus 16 status in cervical swabs of young women with a history of normal Papanicolaou smears. Obstet Gynecol. 1992;79:683-688.

30. Fairley CK, Robinson PM, Chen S, Tabrizi SN, Garland SM. The detec tion of HPV DNA, the size of tampon specimens and the menstrual cycle. Genitourin Med. 1994;70:171-174.

31. Fairley CK, Chen S, Tabrizi S, Quinn M, Garland S. Influence of quartile of menstrual cycle on pellet volume of specimens from tampons and isolation of human papillomavirus. J Infect Dis. 1992;166:1199-1200.

32. Horton NJ, Lipsitz SR. Review of software to fit generalized estimating equation regression models. Am Statist. 1999;53:160-169.

33. Bosch FX, Munoz N, de Sanjose $S$, et al. Risk factors for cervical cancer in Columbia and Spain. Int J Cancer. 1992;52:750-758.

34. Becker TM, Wheeler CM, McGough NS, et al. Contraceptive and reproductive risks for cervical dysplasia in southwestern Hispanic and non-Hispanic white women. Int J Epidemiol. 1994;23:913-922.

35. Kruger-Kjaer S, van den Brule AJ, Svare El, et al. Different risk factor patterns for high-grade and low-grade intraepithelial lesions on the cervix among HPV-positive and HPV-negative young women. Int J Cancer. 1998;76:613-619.

36. Ho GY, Kadish AS, Burk RD, et al. HPV 16 and cigarette smoking as risk factors for high-grade cervical intra-epithelial neoplasia. Int J Cancer. 1998;78:281-285.

37. Murthy NS, Mathew A. Risk factors for pre-cancerous lesions of the cervix. Eur J Cancer Prev. 2000;9:5-14.

38. Wright TC Jr, Cox JT, Massad LS, Twiggs LB, Wilkinson EJ, for the ASCCP-Sponsored Consensus Conference. 2001 Consensus guidelines for the management of women with cervical cytological abnormalities. JAMA. 2002;287:2120-2129. 